ORIGINAL ARTICLE

\title{
Immunostaining patterns of myoepithelial cells in breast lesions: a comparison of CD10 and smooth muscle myosin heavy chain
}

\author{
A N Kalof, D Tam, B Beatty, K Cooper
}

J Clin Pathol 2004;57:625-629. doi: 10.1136/icp.2003.013227

See end of article for authors' affiliations .....................

Correspondence to: Dr A N Kalof, Department of Pathology, Smith Building, Fletcher Allen Health Care-University of Vermont, 111 Colchester Avenue, Burlington, VT 05401, USA; alexandra. kalof@vtmednet.org

Accepted for publication 30 December 2003

\begin{abstract}
Background: Recent studies have reported CD10 expression in myoepithelial cells (MEC) of the breast, supporting its use as a marker to help distinguish invasive breast carcinoma (IC) from ductal carcinoma in situ (DCIS).

Aim: To compare the effectiveness of CDI0 with smooth muscle myosin heary chain (SMMHC) in the detection of MEC in benign and malignant breast lesions.

Methods: Histological material from 25 patients with DCIS and 21 with IC were immunostained for CD 10 and SMMHC. Staining was scored on a scale of 0 to $3+(0$, no staining; $3+$, intense) and the staining distribution was documented as focal, partial, or circumferential.

Results: Uniform, 3+ circumferential CD10 and SMMHC staining of MEC was seen in normal breast ducts and lobules, and in ducts and acini involved in sclerosing adenosis and apocrine metaplasia. In an analysis of total ducts involved by DCIS, 3+ circumferential staining was seen in 65 of 366 ducts (17.7\%) stained for CD10 versus 190 of 396 ducts (48\%) stained for SMMHC. MEC were not detected immunohistochemically in 116 of 366 ducts (31.7\%) with anti-CD10 and 50 of 396 (12.7\%) with antiSMMHC. In contrast, all ICs were negative for both CDIO and SMMHC. Focal background staining of stromal myofibroblasts was seen with both CD10 and SMMHC, but CD10 showed a higher rate of nonspecific staining of epithelial cells.

Conclusion: Although CDIO can aid in the distinction between IC and DCIS, SMMHC is a more sensitive and specific marker of MEC and shows less heterogeneity of immunostaining patterns.
\end{abstract}

M yoepithelial cells (MEC) are contractile elements found in salivary, sweat, and mammary glands that show a combined smooth muscle and epithelial phenotype. ${ }^{1}$ In the normal breast, the ductal and acinar units are lined by two cell layers: the inner layer of epithelial cells lining the lumen and an outer layer of contractile MEC. An intact MEC layer is seen in both benign and in situ lesions, whereas loss of the MEC layer is considered the gold standard for the diagnosis of invasive cancer. ${ }^{2}$

Because MEC are not always readily identifiable on routine haematoxylin and eosin stained sections, many immunohistochemical methods have been used to highlight an intact MEC layer. Given the mixed epithelial and smooth muscle phenotype of MEC, and the need to distinguish the MEC layer from the epithelial cell layer, most of the immunohistochemical markers used are directed against smooth muscle related antigens. These have included antibodies against S-100, ${ }^{34}$ smooth muscle actin (SMA), ${ }^{15-7}$ calponin, ${ }^{178}$ h-caldesmon, ${ }^{18}$ and smooth muscle myosin heavy chain (SMMHC). ${ }^{18}$

\footnotetext{
"An intact myoepithelial cell (MEC) layer is seen in both benign and in situ lesions, whereas loss of the MEC layer is considered the gold standard for the diagnosis of invasive cancer $^{\prime \prime}$
}

SMMHC is a structural component of the smooth muscle myosin molecule and is a specific marker of "terminal" smooth muscle differentiation. ${ }^{2} 89$ SMMHC is composed of at least two isoforms: SMI (204 kDa) and SM2 (200 kDa), both of which are encoded by a single gene. ${ }^{10}{ }^{11}$ The SMl isoform is expressed in the MEC of normal mammary glands, fibrocystic diseases, and in myoepithelial derived tumours of the breast. ${ }^{10}$ Furthermore, studies have documented that antibodies to SMMHC and calponin, both markers of terminal smooth muscle differentiation, are more specific for breast MEC than are other more commonly used antibodies, such as those that recognise SMA. ${ }^{18}$

Recent studies have reported CD10 expression in normal MEC of the breast, ${ }^{512}$ and have demonstrated its usefulness as a breast MEC marker. ${ }^{513} \mathrm{CD} 10$, the common acute lymphoblastic leukaemia antigen, was originally described as a leukaemia associated antigen expressed in lymphoid precursors and germinal B cells, ${ }^{14}{ }^{15}$ and is a useful cell surface marker for the categorisation of acute leukaemias and malignant lymphomas. Recently, an anti-CD10 monoclonal antibody (clone 56C6) has become commercially available for use in formalin fixed, paraffin wax embedded tissues. The expression of this marker has been demonstrated in a wide range of non-haemopoietic tissues, including glomerular cells of the kidney, epithelial cells of the prostate gland and small and large intestine, endometrial stromal cells, ${ }^{12}$ and MEC of the breast. ${ }^{12}{ }^{13} 16$ A recent comparative study of CD10 and SMA expression in MEC of the breast concluded that CDI0 was uniformly positive in MEC of normal breast and may serve as a useful marker of breast MEC in difficult breast lesions (for example, sclerosing adenosis versus tubular carcinoma). ${ }^{5}$

The aim of our study was to evaluate the usefulness of CD10 in the distinction between invasive breast carcinoma and ductal carcinoma in situ (DCIS), and to compare it with

Abbreviations: DCIS, ductal carcinoma in situ; MEC, myoepithelial cells; SMA, smooth muscle actin; SMMHC, smooth muscle myosin heavy chain 
the more frequently used SMMHC. We also examined CDI0 expression in normal breast tissue and benign lesions, including sclerosing adenosis and apocrine metaplasia.

\section{MATERIALS AND METHODS}

Using the SnoMed II system, we searched the Fletcher Allen Health Care surgical pathology archives from July 2000 to June 2003 for excisional breast biopsies with diagnoses of invasive adenocarcinoma (ductal and lobular) and DCIS. Histological material from a total of 31 excisional breast biopsies yielded 25 cases of DCIS and 21 cases of invasive carcinoma ( 12 ductal and nine lobular), some of which were coexistent. The patients were all female and had a mean age of 57.6 years (range, 32-83). Benign elements were evaluated simultaneously and included three cases of sclerosing adenosis and five cases of apocrine metaplasia. The diagnoses of all patients were confirmed by retrieval of pathology reports and review of all haematoxylin and eosin stained sections by an experienced breast pathologist (DT).

Staining for CDI0 was performed using the anti-CD10 monoclonal antibody, clone 56C6 (NCL-CD10-270; NovoCastra, Newcastle upon Tyne, UK). Target retrieval was performed using the Dako (Carpentaria, California, USA) solution of regular sodium citrate, $\mathrm{pH}$ 6.0. Application of the primary antibody at a dilution of $1 / 80$ (table 1) was followed by detection using the avidin-biotin-peroxidase complex technique with diaminobenzidine as the chromagen substrate. Staining for SMMHC (M3558; Dako) was performed at a dilution of $1 / 200$. Normal kidney and breast tissues were used as positive tissue controls for CDI0, and normal breast tissue was used for SMMHC. Isotype matched murine IgG negative controls were run for each specimen.

MEC, benign and malignant epithelial cells, and stromal myofibroblastic elements were evaluated for percentage of elements staining, staining pattern, and intensity. The staining patterns of the ductal MEC were reported as focal $(\leqslant 10 \%$ of duct circumference), partial (10-90\%), or circumferential $(\geqslant 90 \%)$. The staining intensity of the various breast elements was evaluated on a scale from 0 to $3+(0$, no staining; $3+$, intense staining $)$. The number of ducts involved by DCIS in all 25 cases were totalled and scored. The results of each immunohistochemical study were separated into the following groups: $3+$ complete, $3+$ partial, $2+$ complete, 2+ partial, 2+ focal, $1+$ complete, $1+$ partial, $1+$ focal, and 0.

\section{RESULTS}

Table 2 summarises the immunohistochemical results, showing the number of ducts staining positively divided by the total number of ducts involved by DCIS, and the significance of the differences in staining patterns. The following results describe in detail the immunostaining patterns of ducts involved by DCIS analysed by case and by total number of ducts involved.

\section{Ductal carcinoma in situ}

Analysis of cases ( $n=25)$

Staining of the 25 DCIS cases for CD10 revealed six cases with $2+/ 3+$ circumferential staining of MEC in all ducts involved by DCIS. Seventeen cases showed heterogeneous

\begin{tabular}{lllll}
\multicolumn{3}{l}{ Table 1} & \multicolumn{4}{l}{ Source and dilution of the antibodies } \\
\hline Antigen & Clone & Source & Dilution & Cost \\
\hline CD10 & $56 C 6$ & Novocastra & $1: 80$ & $\$ 360 / \mathrm{ml}$ \\
SMMHC & SmmS-1 M3558 & Dako & $1: 200$ & $\$ 240 / \mathrm{ml}$ \\
\hline SMMHC, smooth muscle myosin heavy chain. & \\
\hline
\end{tabular}

staining of the MEC layer: four cases showed weak $(0 / 1+)$ focal to partial staining of all ducts, five cases showed $2+/ 3+$ staining of $10-49 \%$ of ducts, four cases showed $2+/ 3+$ staining of $50-75 \%$ of ducts, and four cases showed $2+/ 3+$ staining of $76-99 \%$ of ducts. In the remaining two cases MEC were negative for CDI0.

Staining of the same cases for SMMHC showed 12 cases with $2+/ 3+$ circumferential staining of MEC in all ducts involved by DCIS. Nine cases showed heterogeneous staining of the MEC layer: three cases showed weak $(0-1+)$ focal staining, one case showed $2+/ 3+$ staining of $10-49 \%$ of ducts, two cases showed $2+/ 3+$ staining of $50-75 \%$ of ducts, and three cases showed $2+/ 3+$ staining of $76-99 \%$ of ducts. Four cases showed no staining of MEC for SMMHC, including one case of micropapillary DCIS arising in an intraductal papilloma.

\section{Analysis of total ducts stained for CD10 ( $\mathrm{n}=366)$ and SMMHC ( $\mathrm{n}=396)$}

Totalling the number of ducts involved by DCIS in all 25 cases, 3+ circumferential CD10 staining was seen in 65 of 366 ducts (17.7\%) compared with 190 of 396 ducts (48\%) stained for SMMHC. MEC were not detected in 116 of 366 ducts $(31.7 \%)$ stained for CD10 and were similarly absent in 50 of 396 ducts $(12.7 \%)$ stained for SMMHC. Within each classification of staining, we compared staining for CD10 with that for SMMHC in the MEC of ducts involved by DCIS. Table 2 shows these results. To determine whether there were significant differences between the two immunohistochemical stains, we conducted Fisher's exact tests on the underlying $2 \times 2$ contingency table (percentage positively stained $v$ percentage negatively stained). All differences between tests that were significant had $p$ values of 0.006 or better.

Thus, the statistical analysis demonstrates that SMMHC and CDIO differ with regard to intensity and distribution of MEC staining. The antibody for SMMHC stains a higher proportion of MEC, with $48 \%$ of cases showing circumferential staining of the MEC layer in all ducts involved by DCIS, compared with only $24 \%$ of cases stained with anti-CDI0.

\section{Invasive carcinoma}

Staining for both CDI0 and SMMHC demonstrated an absence of MEC in all cases of invasive carcinoma ( 12 ductal and nine lobular). Adjacent blood vessels were strongly reactive for SMMHC, serving as good internal controls. In each of the invasive carcinoma cases, there was focal, $1+$ to $2+$ patchy background staining of spindled cells for both CDI0 and SMMHC (fig $1 \mathrm{~A}-\mathrm{C}$ ). These spindled cells were interpreted as myofibroblasts and were associated with the desmoplastic stroma surrounding invasive tumour islands, in addition to the granulation tissue adjacent to previous biopsy sites. The corresponding negative controls did not show immunopositivity of stromal myofibroblasts.

\section{Normal breast elements}

Uniform, 3+ circumferential CDI0 and SMMHC staining of MEC was seen in normal breast ducts and lobules, in addition to ducts and acini involved in sclerosing adenosis (three of three) and apocrine metaplasia (five of five).

\section{DISCUSSION}

Our study found that SMMHC was a better marker than CDI0 for the identification of MEC in breast ducts involved by DCIS. Although CDI0 was consistently expressed in the MEC of normal breast tissue, sclerosing adenosis, and apocrine metaplasia, it showed a heterogeneous staining pattern in ducts involved by DCIS. Specifically, only $32.7 \%$ of ducts stained for CD10 showed complete, strong staining of the MEC layer, and almost a third demonstrated an absence 
Table 2 Immunohistochemical results of DCIS

\begin{tabular}{|c|c|c|c|c|c|c|c|c|c|}
\hline Antigen & $3+$ complete $^{*}$ & $3+$ partial & $2+$ complete & $2+$ partial $^{*}$ & $2+$ focal & $1+$ complete* & $1+$ partial $^{*}$ & $1+$ focal $^{*}$ & 0 * \\
\hline CDI0 & $\begin{array}{l}65 / 366 \\
(17.7 \%)\end{array}$ & $\begin{array}{l}0 / 366 \\
(0 \%)\end{array}$ & $\begin{array}{l}55 / 366 \\
(15.0 \%)\end{array}$ & $\begin{array}{l}20 / 366 \\
(5.5 \%)\end{array}$ & $\begin{array}{l}0 / 351 \\
(0 \%)\end{array}$ & $\begin{array}{l}29 / 366 \\
(7.9 \%)\end{array}$ & $\begin{array}{l}27 / 366 \\
(7.4 \%)\end{array}$ & $\begin{array}{l}54 / 366 \\
(14.8 \%)\end{array}$ & $\begin{array}{l}116 / 366 \\
(31.7 \%)\end{array}$ \\
\hline SMMHC & $\begin{array}{l}190 / 396 \\
(48.0 \%)\end{array}$ & $\begin{array}{l}0 / 379 \\
(0 \%)\end{array}$ & $\begin{array}{l}52 / 396 \\
(13.1 \%)\end{array}$ & $\begin{array}{l}52 / 396 \\
(13.1 \%)\end{array}$ & $\begin{array}{l}3 / 396 \\
(0.8 \%)\end{array}$ & $\begin{array}{l}1 / 396 \\
(0.2 \%)\end{array}$ & $\begin{array}{l}13 / 396 \\
(3.3 \%)\end{array}$ & $\begin{array}{l}35 / 396 \\
(8.8 \%)\end{array}$ & $\begin{array}{l}50 / 396 \\
(12.7 \%)\end{array}$ \\
\hline
\end{tabular}

The values are number of ducts staining positively/total number of ducts involved by DCIS.

*The difference in the proportion of ducts staining positively was significant at $p=0.02$ or better using Fisher's exact test. Other results were not significant at the 0.25 level.

DCIS, ductal carcinoma in situ; SMMHC, smooth muscle myosin heavy chain.

of staining. However, staining for SMMHC was significantly stronger and more complete in MEC of DCIS. Whereas $61.1 \%$ of ducts stained for SMMHC showed complete, $2+$ to $3+$ staining of the MEC layer, only $12.7 \%$ of ducts completely failed to highlight the MEC layer.

Because individual cases showed heterogeneity in MEC staining patterns, which varied considerably between ducts involved by DCIS, the numbers of ducts in all cases were counted and each evaluated individually for intensity and staining pattern. In doing so, we were able to evaluate the characteristics of individual ducts that may have impacted on staining intensity. We found that many ducts that were associated with pronounced inflammation displayed considerably weaker, discontinuous staining for CDl0 (fig 2A-C). The discontinuous pattern of staining also raised the possibility of an intermediate or premalignant state, whereby there is a gradual loss of MEC before stromal invasion.

Because the presence of MEC distinguishes benign from malignant disease, it is important that MEC markers do not crossreact with other cells in the breast, leading to potential misinterpretation. In our study, CDI0 exhibited a reduced specificity for MEC when compared with SMMHC. Although Moritani et al reported no reactivity of CD10 with luminal epithelial cells, ${ }^{5}$ we documented focal staining of luminal epithelial cells of normal ductal epithelium and consistent staining of the luminal surfaces of apocrine metaplastic cells. In addition, there was prominent crossreactivity of anti-CD10 with stromal myofibroblasts. Methodological differences may account for some discrepancies between our study and that reported by Moritani et al, including differences in the antibody clones, antibody titration, and target retrieval.

Interestingly, a recent study of CDI0 expression in colorectal adenomas showed frequent expression of CDI0 by stromal cells in adenomas and invasive carcinomas, ${ }^{17}$ suggesting that CD10 expression may play an integral part in colorectal carcinogenesis. CD10 has also been detected within invasive areas of breast carcinoma, ${ }^{18}{ }^{19}$ supporting its role as a potential participant in the "tumour-stromal interaction". ${ }^{17}$ Iwaya et al reported an increased frequency of stromal expression of CDI0 in invasive breast carcinoma cases with axillary lymph node metastases. ${ }^{19}$ In addition, they correlated stromal CD10 positivity with a shorter time to recurrence, thereby suggesting that stromal expression of CD10 may serve as an important prognostic indicator, a function that would preclude its use as an ideal MEC marker because of interfering background staining. ${ }^{19}$

\section{"Because no myoepithelial cell marker to date exhibits perfect sensitivity and specificity, it is recommended that a combination of immunohistochemical stains be used when investigating difficult breast lesions"}

Anti-SMMHC also showed background staining of stromal myofibroblasts, albeit to a lesser degree. Given that myofibroblasts and MEC share a mixed smooth muscle phenotype, antibodies to many markers of smooth muscle differentiation (such as SMA) have shown reduced specificity, with background staining of myofibroblasts. SMMHC has been regarded as a marker of terminal smooth muscle differentiation, and has demonstrated higher specificity for MEC compared with myofibroblasts in many studies. ${ }^{20}$ Positive staining of stromal myofibroblasts for SMMHC has nevertheless been documented previously in breast cancers, ${ }^{21}$ and our study shows significant crossreactivity with stromal myofibroblasts. Method comparisons show that these studies have used the same SMMS-1 antibody clone with similar dilutions, varying from $1 / 20^{9}$ to $1 / 60 .^{20}$ We are unable to account for the differences in crossreactivity, indicating that some cases need to be interpreted with caution because the positively staining myofibroblasts abutting infiltrating carcinoma may be misinterpreted as MEC, resulting in a potential misdiagnosis of in situ carcinoma.
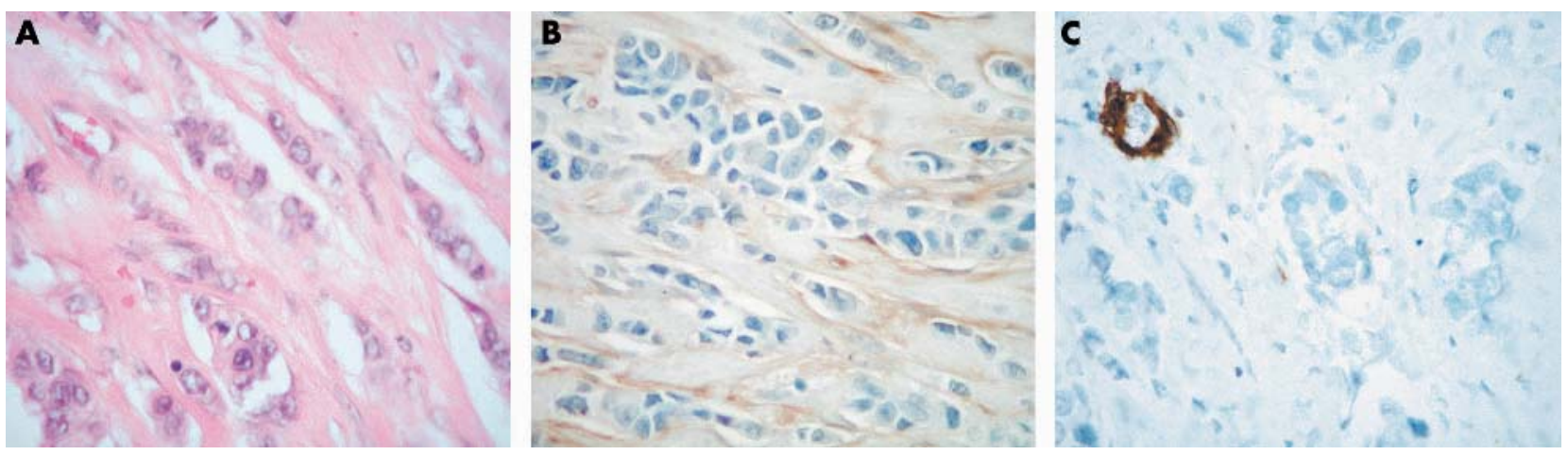

Figure 1 (A) Invasive ductal adenocarcinoma (haematoxylin and eosin stain; original magnification, $\times 400$ ). (B) Invasive ductal adenocarcinoma. Note the positively staining background myofibroblasts (staining for CD10; original magnification, $\times 400$ ). (C) Invasive ductal adenocarcinoma. Note the reduced staining of background stromal cells but positively staining blood vessels, serving as good internal controls for smooth muscle myosin heavy chain $(S M M H C)$ (staining for SMMHC; original magnification, $\times 400)$. 

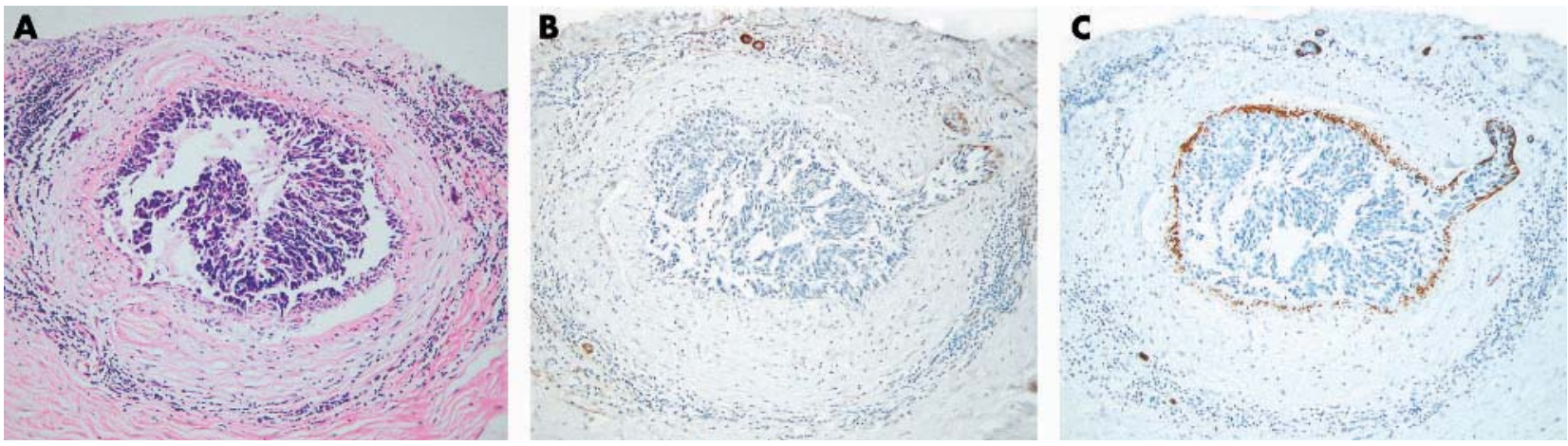

Figure 2 (A) Focus of ductal carcinoma in situ (DCIS) with surrounding inflammation (haematoxylin and eosin stain; original magnification, $\times 200$ ). (B) Immunohistochemistry for CD10 on the same focus of DCIS. Note the lack of a positively staining myoepithelial cell (MEC) layer (CD10; original magnification, $\times 200)$. (C) Immunohistochemistry for smooth muscle myosin heavy chain (SMMHC) on the same focus of DCIS with strong staining of the MEC layer (SMMHC; original magnification, $\times 200)$.

There are other factors suggesting that staining for SMMHC is superior to staining for CD10 for the identification of MEC in breast samples. For example, similar to other studies, ${ }^{2}$ we found that optimum staining for CDI0 required the antibody to be diluted $1 / 80$ compared with $1 / 200$ for SMMHC. In addition, there is a considerable cost differential between the two antibodies (in our case, $\$ 360 / \mathrm{ml}$ for antiCDI0 $v \$ 240 / \mathrm{ml}$ for anti-SMMHC), indicating that SMMHC is more cost effective for routine use in the laboratory.

More sensitive and specific markers of MEC are constantly being sought to help pathologists with difficult breast lesions. p63, a recently identified member of the p53 gene family, has been found to be another reliable and sensitive marker of MEC of the breast..$^{22}$ Of particular interest, Barbareschi et al showed that the background myofibroblastic cells were consistently non-reactive with anti-p63. ${ }^{22}$ A more recent study by Werling et al compared the usefulness of p63 with calponin and SMMHC for identifying MEC in breast tissue. They found that although p63 offers excellent sensitivity and increased specificity for MEC, the antibody to p63 reacted with a small proportion ( $11 \%)$ of breast carcinoma tumour cells. In addition, anti-p63 occasionally demonstrated a discontinuous MEC layer surrounding nests of DCIS. Werling et al recommend that expression of p63 should be used in conjunction with SMMHC for the identification of MEC in difficult breast lesions. ${ }^{20}$

In conclusion, although expression of CD10 may aid in the distinction between DCIS and invasive breast carcinoma, SMMHC exhibits increased sensitivity and specificity for MEC and is more cost effective for routine use in identification of MECs of the breast. We have found that staining for

\section{Take home messages}

- Although CD10 expression can aid in the distinction between invasive breast carcinoma and ductal carcinoma in situ, smooth muscle myosin heavy chain (SMMHC) is a more sensitive and specific marker of myoepithelial cells and shows less heterogeneity of immunostaining patterns

- In addition, staining for SMMHC is more cost effective than staining for CD10

- Because none of the myoepithelial markers shows perfect sensitivity and specificity, a combination of immunohistochemical stains should be used when investigating difficult breast lesions
SMMHC has worked well in our laboratory and use it routinely in isolation for the detection of breast MEC. However, because no MEC marker to date exhibits perfect sensitivity and specificity, it is recommended that a combination of immunohistochemical stains be used when investigating difficult breast lesions.

\section{ACKNOWLEDGEMENTS}

We thank H Aliesky and L Simmons-Arnold for technical support and Drs L Kalof and T Dietz for comments on earlier drafts. This research was funded by the Division of Experimental Pathology at the University of Vermont.

\section{Authors' affiliations}

A N Kalof, D Tam, B Beatty, K Cooper, University of Vermont-Fletcher Allen Health Care, Burlington, Vermont, VT 05401, USA

\section{REFERENCES}

1 Foschini MP, Scarpellini F, Gown AM. Differential expression of myoepithelial markers in salivary, sweat and mammary glands. Int J Surg Pathol 2000;8:29-37.

2 Yaziii H, Gown AM, Sneige. Detection of stromal invasion in breast cancer: the myoepithelial markers. Adv Anat Pathol 2000;7:100-9.

3 Egan MJ, Newman J, Crocker J, et al. Immunohistochemical localization of S100 protein in benign and malignant conditions of the breast. Arch Pathol Lab Med 1987;111:28-31

4 Hijazi YM, Lessard JL, Weiss MA. Use of anti-actin and S100 protein antibodies in differentiating benign and malignant sclerosing breast lesions. Surg Pathol 1989:2:125-35.

5 Moritani S, Kushima R, Sugihara H, et al. Availability of CD10 immunohistochemistry as a marker of breast myoepithelial cells on paraffin sections. Mod Pathol 2002; 15:397-405.

6 Gugliotta P, Sapino A, Macri L, et al. Specific demonstration of myoepithelial cells by anti-alpha smooth muscle actin antibody. J Histochem Cytochem, 1988; 36:659-63

7 Daminani S, Ludvikova M, Tomasic G, et al. Myoepithelial cells and basal lamina in poorly differentiated in situ duct carcinoma of the breast: an immunocytochemical study. Virchows Arch 1999:434:227-34.

8 Wang NP, Wan BC, Gown AM, et al. Antibodies to novel myoepitheliumassociated proteins distinguish benign lesions and carcinoma in situ from invasive carcinoma of the breast. Appl Immunohistochem 1997:5:141-51.

9 Longtine JA, Pinkus GS, Corson JM, et al. Immunohistochemical localization of smooth muscle myosin in normal human tissues. J Histochem Cytochem 1985;33:179-84

10 Ohyabu I, Takasaki T, Yoshida $\mathrm{H}$, et al. Immunohistochemical studies on expression of human vascular smooth muscle myosin heavy chain isoforms in normal mammary glands, benign mammary disorders and mammary carcinomas. Pathol Int 1998;48:433-9.

11 Babu GJ, Warshaw DM, Periasamy M. Smooth muscle myosin heavy chain isoforms and their role in muscle physiology. Microsc Res Tech 2000:50:532-40.

12 Chu P, Arber D. Paraffin-section detection of CD 10 in 505 nonhematopoietic neoplasms: frequent expression in renal cell carcinoma and endometrial stromal sarcoma. Am J Clin Pathol 2000;113:374-82.

13 Mahendran R, Mcllhinney R, O'Hare M, et al. Expression of the common acute lymphoblastic leukaemia antigen (CALLA) in the human breast. Mol Cell Probes 1989;3:39-44 
14 Greaves MF, Janossy J. Patterns of gene expression and the cellular origins of human leukemias. Biochem Biophys Acta 1978;516:193.

15 Carrel S, Schmidt-Kessen A, Mach JP, et al. Expression of common acute lymphoblastic leukemia (CALLA) by lymphomas of $B$ and $T$ cell lineage. J Immunol 1983;130:2456-60.

16 Metzgar RS, Borowitz MJ, Jones NH, et al. Distribution of common acute lymphoblastic leukemia antigen in nonhematopoietic tissues. J Exp Med 1981;154:1249-54.

17 Ogawa H, Iwaya K, Mukai K, et al. Expression of CD10 by stromal cells during colorectal tumor development. Hum Pathol 2002;33:806-11.

18 Atherton AJ, O'Hare MJ, Buluwela L, et al. Ectoenzyme regulation by phenotypically distinct fibroblast sub-populations isolated from the human mammary gland. J Cell Sci 1994;107:2931-9.
19 Iwaya K, Ogawa H, Izumi M, et al. Stromal expression of CD10 in invasive breast carcinoma: a new predictor of clinical outcome. Virchows Arch 2002;440:589-93.

20 Werling RW, Hwang $\mathrm{H}$, Yaziji $\mathrm{H}$, et al. Immunohistochemical distinction of invasive from noninvasive breast lesions: a comparative study of p63 versus calponin and smooth muscle myosin heavy chain. Am J Surg Pathol 2003;27:82-90.

21 Chiavegato A, Bochaton-Piallat ML, Gabbiani G, et al. Expression of myosin heavy chain isoforms in mammary epithelial cells and in myofibroblasts from different fibrotic settings during neoplasia. Virchows Arch 1995;426:77-86.

22 Barbareschi M, Pecciarini L, Doglioni C, et al. p63, a p53 homologue, is a selective nuclear marker of myoepithleial cells of the human breast. Am J Surg Pathol 2001;25:1054-60. 